\title{
F(E,L $\mathrm{L}_{\mathrm{Z}}$ FOR KENT'S MODEL OF THE GALACTIC BULGE
}

\author{
WALTER DEHNEN \\ Theoretical Physics \\ Oxford, OX1 3NP \\ United Kingdom
}

\begin{abstract}
Using the Richardson-Lucy algorithm the two-integral distribution function (2I-DF) $f\left(E, L_{z}\right)$ for Kent's (1992) Bulge model has been constructed. It turns out to be negative (and hence unphysical) at $L_{z} \approx L_{\mathrm{c}}$. The physical reason for this result and its implications are discussed.
\end{abstract}

The even part $f_{\mathrm{e}}\left(E, L_{z}\right)$ of the 2I-DF that creates the axisymmetric density $\rho(R, z)$ is uniquely determined by $\rho(R, z)$ and the potential, but the analytic inversion formulae (e.g. Hunter \& Qian 1993) require the knowledge of density and potential outside the physical domain (at complex $z$ ), and are numerically unstable, i.e. noise in $\rho$ is amplified into noise in the DF. However, the Richardson (1972) -Lucy (1974) algorithm can be used to fast and accurately compute $f_{\mathrm{e}}\left(E, L_{z}\right)$ for any axisymmetric stellar system, as I showed in Dehnen (1994), where M32 is modeled. Here this method is applied to Kent's (1992) mass model for the Bulge, which consists of a $s^{-1.85}$ cusp inside $0.9 \mathrm{kpc}$ and falls off steeply outside, where

$$
s^{m}=R^{m}+(z / 0.61)^{m}, \quad m=4 .
$$

The potential is that of Bulge and disk. The 2I-DF comes out to be negative at high angular momenta for all energies. This is independent of the disk, which contributes only marginally to the potential inside the cusp, and of the presence of a central black hole, which affects only the innermost $10 \mathrm{pc}$.

In order to understand this result, consider the run of density along a potential contour, which for $f=f(E)$ simply is a constant. In case of a boxy density the potential will be rounder, and the "corner" of the boxy $\rho$-contours creates a peak in the run of $\rho$ along a $\Psi$-contour, see the Fig. 1 . To create this maximum, the $2 \mathrm{I}-\mathrm{DF}$ has to strongly populate orbits with intermediate $L_{z} / L_{\mathrm{c}}(E)$. However, these orbits also contribute to, and even 

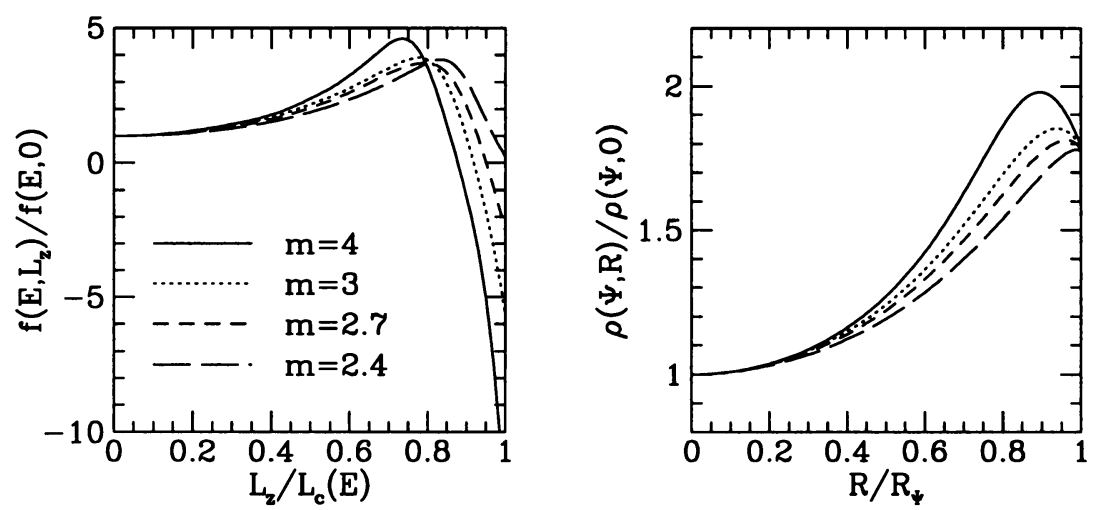

Figure 1. Left: run of the 2I-DF with $L_{z}$ at the energy of the circular orbit at 100pc. Right: run of $\rho$ along the potential contour of $\Psi(R=100 \mathrm{pc}, z=0)$. The lines correspond to different values of $m$ in eq. (1), $m=4$ gives Kent's (1992) model.

overpopulate the density on the equator. Therefore, to gain the correct density there, $f_{\mathrm{e}}\left(E, L_{z}\right)$ has to become negative for near-circular orbits. This is illustrated by Fig. 1 which shows $f_{\mathrm{e}}\left(E, L_{z}\right)$ vs. $L_{z}$ at fixed $E$ (left) and $\rho$ along a potential contour (right).

The outcome of a negative 2I-DF is an example for the danger in using the Jeans equations for galaxy modeling, which do not tell anything about the physicality of the underlying DF. Kuijken (1994) also found indications for negativity of the 2I-DF for Kent's Bulge model. Another hint comes from Evans' (1994) power-law models, which have spheroidal potentials and boxy densities. Using his model with $\rho \propto r^{-1.85}$, one finds that the 2I-DF is non-physical for axis ratios less than 0.65 (Kent's models has 0.61 ).

There are three possibilities, to alter the used mass distribution in order to gain a model with physical 2I-DF. As indicated by Evans' (1994) models, a greater axis ratio (i) or a shallower cusp (ii) will certainly help. A less boxy density (iii) should also alleviate the problem. Experiments with smaller powers $m$ in eq. (1) showed, that for a modest boxiness with $m=2.4$ the 2I-DF is just physical (see Fig. 1). However, physical 2I-DFs with peanutshaped densities exist, which indicates, that Kent's parametrisation of the boxyness (eq. 1) is a bad choice, as it cannot create a peanut shape.

\section{References}

Dehnen W., 1994, MNRAS, submitted

Evans N.W., 1994, MNRAS 267, 333

Hunter C., Qian E., 1993, MNRAS 262, 401

Kent S.M., 1992, ApJ 387, 181

Kuijken K., 1994, preprint

Lucy L.B., 1974, AJ 79, 745

Richardson W.H., 1972, J. Opt. Soc. Am. 62, 55 